\title{
A New Relativistic Study for Interactions in One-electron atoms (Spin 1/2 Particles) with Modified Mie-type Potential
}

\author{
Abdelmadjid Maireche* \\ Laboratory of Physics and Material Chemistry, Physics department, Sciences Faculty, \\ University of M'sila-M'sila, Algeria
}

(Received 08 June 2016; revised manuscript received 22 November 2016; published online 29 November 2016)

In this paper, we present a novel theoretical analytical perform further investigation for the exact solvability of relativistic quantum spectrum systems for modified Mie-type potential (m.m.t.) potential is discussed for spin-1/2 particles by means Boopp's shift method instead to solving deformed Dirac equation with star product, in the framework of noncommutativity three dimensional real space (NC: 3D-RS). The exact corrections for excited $n^{\text {th }}$ states are found straightforwardly for interactions in one-electron atoms by means of the standard perturbation theory. Furthermore, the obtained corrections of energies are depended on four infinitesimal parameter $\Theta$,which induced by position-position noncommutativity, in addition to the discreet atomic quantum numbers: $j=\tilde{l} \pm 1 / 2, s= \pm 1 / 2, \tilde{l}$ and $\tilde{m}$ (the angular momentum quantum number) and we have also shown that, the usual states in ordinary two and three dimensional spaces are canceled and has been replaced by new degenerated $2(2 \tilde{l}+1)$ sub-states in the new quantum symmetries of (NC: 3D-RS) and we have also applied our obtained results to the case of modified KrazerFutes potential.

Keywords: The Mie-type potential, Krazer-Futes potential, Noncommutative space, Star product and Boopp's shift method and Dirac.

DOI: $10.21272 /$ jnep.8(4(1)).04027

PACS numbers: 03.65.Pm, 03.65.Ge

\section{INTRODUCTION}

Fifty years ago, a big scientific revolution successful for describing atoms, nuclei, and molecules and their spectral behaviors based on three fundamental equations: Schrödinger (spin $1 / 2$ particle at low energy) [126], Klein-Gordon (spin zero particle at high energy) and Dirac (spin $1 / 2$ particle at high energy) [27-37]. Dirac equation with physically significant potentials and the latest in terms of interest, it is playing a crucial role in modern quantum mechanics, many potentials are treated within the framework of relativistic quantum mechanics based on this equation in two, three and $D$ generalized spaces, the quantum structure based to the ordinary canonical commutations relations (CCRs) in both Schrödinger and Heisenberg (the operators are depended on time) pictures, respectively, as $(c=\hbar=1)$ :

$$
\begin{gathered}
{\left[x_{i}, p_{j}\right]=i \delta_{i j} \text { and }} \\
{\left[x_{i}, x_{j}\right]=\left[p_{i}, p_{j}\right]=0} \\
{\left[x_{i}(t), p_{j}(t)\right]=i \delta_{i j} \text { and }} \\
{\left[x_{i}(t), x_{j}(t)\right]=\left[p_{i}(t), p_{j}(t)\right]=0}
\end{gathered}
$$

where the two operators $\left(x_{i}(t), p_{i}(t)\right)$ in Heisenberg picture are related to the corresponding two operators $\left(x_{i}, p_{i}\right)$ in Schrödinger picture from the two projections relations:

$$
\begin{aligned}
& x_{i}(t)=\exp \left(i \hat{H}\left(t-t_{0}\right)\right) x_{i} \exp \left(-i \hat{H}\left(t-t_{0}\right)\right) \\
& p_{i}(t)=\exp \left(i \hat{H}\left(t-t_{0}\right)\right) p_{i} \exp \left(-i \hat{H}\left(t-t_{0}\right)\right)
\end{aligned}
$$

Here $\hat{H}$ denote to the ordinary quantum Hamiltonian operator. In addition, for spin $1 / 2$ particles described by the Dirac equation, experiment tells us that must satisfy Fermi Dirac statistics obey the restriction of Pauli, which imply to gives the only non-null equaltime anti-commutator for field operators as follows:

$$
\left\{\Psi_{\alpha}(t, \mathrm{r}), \bar{\Psi}_{\beta}\left(t, \mathrm{r}^{\prime}\right)\right\}=i \gamma_{\alpha \beta}^{0} \delta^{3}\left(\mathrm{r}-\mathrm{r}^{\prime}\right)
$$

with $\bar{\Psi}_{\beta}\left(t, \mathrm{r}^{\prime}\right)=\Psi_{\beta}^{+}\left(t, \mathrm{r}^{\prime}\right) \gamma^{0}$. H. Snyder it was first who introduce the noncommutativity idea for almost seventy years ago [38], recently, much considerable effort has been expanded on the solutions of Schrödinger, Dirac and Klein-Gordon equations to noncommutative quantum mechanics [39-72], the new quantum structure of noncommutative space based on the following noncommutative canonical commutations relations (NCCRs) in both Schrödinger and Heisenberg pictures, respectively, as follows [39-72]:

$$
\begin{aligned}
& {\left[\hat{x}_{i}^{*}, \hat{p}_{j}\right]=i \delta_{i j},\left[\hat{x}_{i}^{*}, \hat{x}_{j}\right]=i \theta_{i j} \quad \text { and } \quad\left[\hat{p}_{i}^{*}, \hat{p}_{j}\right]=0} \\
& {\left[\hat{x}_{i}(t)^{*}, \hat{p}_{j}(t)\right]=i \delta_{i j},\left[\hat{x}_{i}(t)^{*}, \hat{x}_{j}(t)\right]=i \theta_{i j} \text { and }} \\
& {\left[\hat{p}_{i}(t)^{*}, \hat{p}_{j}(t)\right]=0}
\end{aligned}
$$

abmaireche@gmail.com 
Where the two new operators $\left(\hat{x}_{i}(t), \hat{p}_{i}(t)\right)$ in Heisenberg picture are related to the corresponding two new operators $\left(\hat{x}_{i}, \hat{p}_{i}\right)$ in Schrödinger picture from the two projections relations:

$$
\begin{aligned}
& \hat{x}_{i}(t)=\exp \left(i \hat{H}_{n c}\left(t-t_{0}\right)\right) * \hat{x}_{i}{ }^{*} \exp \left(-i \hat{H}_{n c}\left(t-t_{0}\right)\right) \\
& \hat{p}_{i}(t)=\exp \left(i \hat{H}_{n c}\left(t-t_{0}\right)\right){ }^{*} \hat{p}_{i}{ }^{*} \exp \left(-i \hat{H}_{n c}\left(t-t_{0}\right)\right)
\end{aligned}
$$

Here $\hat{H}_{n c}$ denote to the new quantum Hamiltonian operator in the symmetries of (NC: 3D-RS). The very small parameters $\theta^{\mu \nu}$ (compared to the energy) are elements of antisymmetric real matrix and $(*)$ denote to the new star product, which is generalized between two arbitrary functions $f(x) \rightarrow \hat{f}(\hat{x})$ and $g(x) \rightarrow \hat{g}(\hat{x})$ to $\hat{f}(\hat{x}) \hat{g}(\hat{x}) \equiv(f * g)(x)$ instead of the usual product $(f g)(x)$ in ordinary three dimensional spaces [41-57]:

$$
\begin{aligned}
\hat{f}(\hat{x}) \hat{g}(\hat{x}) & \equiv(f * g)(x) \equiv \exp \left(\frac{i}{2} \theta^{\mu v} \partial_{\mu}^{x} \partial_{v}^{x}(f g)(x, p)\right. \\
& \equiv\left(f g-\left.\frac{i}{2} \theta^{\mu v} \partial_{\mu}^{x} \partial_{v}^{x} g\right|_{\left(x^{\mu}=x^{\nu v}\right)}+O\left(\theta^{2}\right)\right.
\end{aligned}
$$

where $\hat{f}(\hat{x})$ and $\hat{g}(\hat{x})$ are the new function in (NC: 3DRS), $\partial_{\mu}^{x} f(x)$ denotes to the $\frac{\partial f(x)}{\partial x^{\mu}}$, the following term $\left(-\frac{i}{2} \theta^{\mu v} \partial_{\mu}^{x} f(x) \partial_{\nu}^{x} g(x)\right)$ is induced by (space-space) noncommutativity properties and $O\left(\theta^{2}\right)$ stands for the second and higher order terms of $\theta$, a Boopp's shift method can be used, instead of solving any quantum systems by using directly star product procedure [3971]:

$$
\left[\hat{x}_{i}, \hat{x}_{j}\right]=i \theta_{i j} \text { and }\left[\hat{p}_{i}, \hat{p}_{j}\right]=0
$$

The three-generalized coordinates $\left(\hat{x}=\hat{x}_{1}, \hat{y}=\hat{x}_{2}, \hat{z}=\hat{x}_{3}\right)$ in the noncommutative space are depended with corresponding three-usual generalized positions $(x, y, z)$ and momentum coordinates $\left(p_{x}, p_{y}, p_{z}\right)$ by the following relations, as follows $[48,49,53,54,58,61]$ :

$$
\begin{aligned}
& \hat{x}=x-\frac{\theta_{12}}{2} p_{y}-\frac{\theta_{13}}{2} p_{z}, \quad \hat{y}=y-\frac{\theta_{21}}{2} p_{x}-\frac{\theta_{23}}{2} p_{z} . \\
& \hat{z}=z-\frac{\theta_{31}}{2} p_{x}-\frac{\theta_{32}}{2} p_{y}
\end{aligned}
$$

The non-vanish -commutators in (NC-3D: RS) can be determined as follows:

$$
\begin{aligned}
& {\left[\hat{x}, \hat{p}_{x}\right]=\left[\hat{y}, \hat{p}_{y}\right]=\left[\hat{z}, \hat{p}_{z}\right]=i,} \\
& {[\hat{x}, \hat{y}]=i \theta_{12},[\hat{x}, \hat{z}]=i \theta_{13},[\hat{y}, \hat{z}]=i \theta_{23}}
\end{aligned}
$$

which allow us to getting the operator $\hat{r}^{2}$ on noncommutative three dimensional spaces as follows $[48,49,53,54,58,61]$ :

$$
\hat{r}^{2}=r^{2}-\overrightarrow{\mathbf{L}} \vec{\Theta}
$$

Where the coupling $\mathbf{L} \Theta$ is given by $\left(\Theta_{i j}=\frac{\theta_{i j}}{2}\right)$ :

$$
\mathbf{L} \Theta \equiv L_{x} \Theta_{12}+L_{y} \Theta_{23}+L_{z} \Theta_{13}
$$

with:

$$
L_{x}=y p_{z}-z p_{y}, L_{y}=\mathrm{zp}_{\mathrm{x}}-\mathrm{xp}_{z} \text { and } L_{z}=x p_{y}-y p_{x}
$$

Furthermore, the non-null equal-time anticommutator for fermionic field operators in noncommutative spaces can be expressed in the following postulate relations:

$$
\begin{aligned}
& \left\{\hat{\Psi}_{\alpha}(t, \mathrm{r}),{ }^{*} \hat{\bar{\Psi}}_{\beta}\left(t, \mathrm{r}^{\prime}\right)\right\}=i \gamma_{\alpha \beta}^{0} \delta^{3}\left(\mathrm{r}-\mathrm{r}^{\prime}\right) \\
& \left\{\hat{\Psi}_{\alpha}(t, \mathrm{r}),{ }^{*}, \hat{\Psi}_{\alpha}\left(t, \mathrm{r}^{\prime}\right)\right\}=\left\{\hat{\bar{\Psi}}_{\alpha}(t, \mathrm{r}){ }^{*}, \hat{\bar{\Psi}}_{\beta}\left(t, \mathrm{r}^{\prime}\right)\right\}=i \theta_{\alpha \beta} \delta^{3}\left(\mathrm{r}-\mathrm{r}^{\prime}\right)
\end{aligned}
$$

And the noncommutative fermion propagator $S_{F}\left(x-x^{\prime}\right)$ :

$$
\begin{aligned}
& S_{F}\left(x-x^{\prime}\right)=-i\left\langle T\left(\Psi(x) * \bar{\Psi}\left(x^{\prime}\right)\right)\right\rangle= \\
& = \begin{cases}\Psi(x)^{*} \bar{\Psi}\left(x^{\prime}\right) & \text { if } \mathrm{t}\rangle \mathrm{t}^{\prime} \\
\bar{\Psi}\left(x^{\prime}\right) * \Psi(x) & \text { if } \left.\mathrm{t}^{\prime}\right\rangle \mathrm{t}\end{cases}
\end{aligned}
$$

$\mathrm{T}$ is the time-ordered product. In particularly, the study of Mie-type potential has now become a very interest field due to their applications in different fields, this potentials have the general features of the true interaction energy, inter atomic and dynamical properties in solid-state physics and play an important role in the history of molecular structures and interactions [24-25, 36-37], this work is aimed at obtaining an analytic expression for the eigenenergies of a modified Mietype potential (m.m.t.) potential in (NC: 3D-RS) using the generalization Boopp's shift method to discover the new symmetries and a possibility to obtain another applications to this potential in different fields. This work based essentially on our previously works [39-58]. The organization scheme of the study is given as follows: In next section, we briefly review the Dirac equation with Mie-type potential on based to Refs. [36-37]. The Section 3, devoted to studying the three deformed Dirac equation by applying Boopp's shift method. In the fourth section and by applying standard perturbation theory we find the quantum spectrum of the $n^{\text {th }}$ excited states in (NC-3D: RS) for relativistic spin-orbital interaction. In the next section, we derive the magnetic spectrum for studied potential. In the sixth section, we resume the global spectrum and corresponding noncommutative Hamiltonian for (m.m.t.) potential. Finally, the important results and the conclusions are discussed in last section. 


\section{REVIEW THE DIRAC EQUATION FOR EX- TENDED MIE-TYPE POTENTIAL IN THREE DIMENSIONAL SPAPACES}

In this section, we shall review the eigenfunctions and eigenvalues for spherically symmetric for the potential $V_{\text {Mie }}(r)$ and the tensor interaction $U(r)$ [3637]:

$$
V_{\text {Mie }}(r)=\frac{a}{r^{2}}-\frac{b}{r}+c \quad \text { and } \quad U(r)=-\frac{T}{r}
$$

where $\left(Z_{a}, Z_{b}\right)$ are charges of projectile particle and target particle,$(a, b, c)$ are arbitrary positive constants and $T=\frac{Z_{a} Z_{b} e^{2}}{4 \prod \varepsilon_{0}}$. The Dirac equation in the presence of above interactions [36-37]:

$$
\begin{aligned}
& (\alpha \mathrm{P}+\beta(M+S(r))-i \beta \hat{r} U(r) \alpha) \Psi(r, \theta, \phi)= \\
& =(E-V(r)) \Psi(r, \theta, \phi)
\end{aligned}
$$

here $M, E$ and $\left(\alpha_{i}=\left(\begin{array}{cc}0 & \sigma_{i} \\ \sigma_{i} & 0\end{array}\right), \beta=\left(\begin{array}{cc}I_{2 \times 2} & 0 \\ 0 & I_{2 \times 2}\end{array}\right)\right)$ are where the fermions' mass, the relativistic energy and the usual Dirac matrices, the spinor $\Psi(r, \theta, \phi)$ :

$$
\Psi_{n k}(r, \theta, \phi)=\left(\begin{array}{c}
f_{n k}(\vec{r}) \\
g_{n k}(\vec{r})
\end{array}\right)=\frac{1}{r}\left(\begin{array}{l}
F_{n k}(r) Y_{j m}^{l}(\theta, \phi) \\
i G_{n \tilde{k}}(r) Y_{j m}^{\tilde{l}}(\theta, \phi)
\end{array}\right)
$$

where $\sigma_{1}=\left(\begin{array}{ll}0 & 1 \\ 1 & 0\end{array}\right), \sigma_{2}=\left(\begin{array}{cc}0 & -i \\ i & 0\end{array}\right)$ and $\sigma_{3}=\left(\begin{array}{cc}1 & 0 \\ 0 & -1\end{array}\right)$ and are $2 \times 2$ three Pauli matrices while $k(\tilde{k})$ is related to the total angular momentum quantum numbers for spin symmetry $l$ and p-spin symmetry $\tilde{l}$ as [36-37]:

$$
k=\left\{\begin{array}{l}
-(l+1) \text { if }-(\mathrm{j}+1 / 2),\left(\mathrm{s}_{1 / 2}, p_{3 / 2}, \text { etc }\right), j=l+\frac{1}{2}, \\
\text { aligned } \operatorname{spin}(\mathrm{k}\langle 0) \\
+l \quad \text { if } j=l+\frac{1}{2},\left(\mathrm{p}_{1 / 2}, d_{3 / 2}, \text { etc }\right), j=l-\frac{1}{2}, \\
\text { unaligned } \operatorname{spin}(\mathrm{k}\rangle 0)
\end{array}\right.
$$

and

$$
\tilde{k}=\left\{\begin{array}{l}
-\tilde{l} \quad \text { if }-(\mathrm{j}+1 / 2),\left(\mathrm{s}_{1 / 2}, p_{3 / 2}, \text { etc }\right), j=\tilde{l}-\frac{1}{2}, \\
\text { aligned } \operatorname{spin}(\mathrm{k}\langle 0) \\
+(\tilde{l}+1) \quad \text { if } j=\tilde{l}+\frac{1}{2},\left(\mathrm{p}_{1 / 2}, d_{3 / 2}, \text { etc }\right), j=\tilde{l}+\frac{1}{2}, \\
\text { unaligned spin }(\mathrm{k}\rangle 0)
\end{array}\right.
$$

The radial functions $\left(F_{n k}(r), G_{n k}(r)\right)$ are obtained by solving the following differential equations [36-37]:

$$
\begin{aligned}
& {\left[\frac{d^{2}}{d r^{2}}-\frac{k(k+1)}{r^{2}}+\frac{2 k}{r} U(r)-\frac{d U(r)}{d r}-U^{2}(r)\right] F_{n k}(r)} \\
& =\left[M+E_{n k}-V(r)\right]\left[M-E_{n k}+C_{p s}\right] F_{n k}(r) \\
& {\left[\frac{d^{2}}{d r^{2}}-\frac{k(k-1)}{r^{2}}+\frac{2 k}{r} U(r)+\frac{d U(r)}{d r}-U^{2}(r)\right] G_{n \tilde{k}}(r)} \\
& =\left[M+E_{n k}-V(r)\right]\left[M-E_{n k}+C_{p s}\right] G_{n \tilde{k}}(r)
\end{aligned}
$$

According to The Laplace transform approach (LTA) and asymptotic interaction method, which applied in refs. [36-37], the upper spinor $F_{n k}(r)$ from relation:

$$
\begin{aligned}
& F_{n k}(r)=\sqrt{\frac{n !(2 \mathrm{n}+2 \gamma+1)}{\Gamma(\mathrm{n}+2 \gamma+1)}} \frac{\Gamma(2 \gamma+1)}{\left(M-E_{n k}+C_{p s}\right)}\left(\frac{d}{d r}-\frac{k+T}{r}\right) \times \\
& \times r^{\gamma+1 / 2} e^{-\varepsilon r}{ }_{1} F_{1}(-n, 2 \gamma+1,2 \varepsilon r)
\end{aligned}
$$

where ${ }_{1} F_{1}(-n, 2 \gamma+1,2 \varepsilon r)$ the confluent hyper-geometric functions and $M \neq E_{n k}$ when $C_{p s}=0$, which means that only negative energy spectrum is permissible for a normalizable and well-defined wave function and in the exact $p$-spin limit and in the absence of tensor interaction, we have the energy equation [36-37]:

$$
\begin{aligned}
& \frac{b\left(E_{n k}-M-C_{p s}\right)}{\sqrt{\left(M+E_{n k}-c\right)\left(M-E_{n k}+C_{p s}\right)}}= \\
& =1+2 n+2 \sqrt{(k+T-1 / 2)^{2}+a\left(E_{n k}-M-C_{p s}\right)}
\end{aligned}
$$

$n=0,1,2 \ldots$ In the exact $p$-spin limit and in the absence of tensor interaction, we have [36]:

$$
\begin{aligned}
& G_{n k}(r)=\Gamma(2 \gamma+1) \sqrt{\frac{n !(2 \mathrm{n}+2 \gamma+1)}{\Gamma(\mathrm{n}+2 \gamma+1)}} \times \\
& \times r^{\gamma+1 / 2} e^{-\varepsilon r}{ }_{1} F_{1}(-n, 2 \gamma+1,2 \varepsilon r)
\end{aligned}
$$

And

$$
\begin{aligned}
& \frac{b\left(E_{n k}-M\right)}{\sqrt{M^{2}-E_{n k}^{2}+c\left(E_{n k}-M\right)}}= \\
& =2 \sqrt{(k-1 / 2)^{2}+a\left(E_{n k}-M\right)}+n+1 / 2
\end{aligned}
$$

With

$$
\begin{aligned}
& \gamma=\sqrt{(k-1 / 2)^{2}+a\left(E_{n k}-M\right)} \text { and } \\
& \varepsilon=\sqrt{M^{2}-E_{n k}^{2}+c\left(E_{n k}-M\right)}
\end{aligned}
$$

The generalized Laguerre polynomials $L_{n}^{(\beta)}(r)$ can be expressed as a function of the confluent hypergeometric functions as [37, 73-74]:

$$
L_{n}^{(p)}(x)=\frac{\Gamma(n+p+1)}{n ! \Gamma(p+1)}{ }_{1} F_{1}(-n, p+1 ; x)
$$


Which allow us to rewritten eq. (12) as:

$$
\begin{aligned}
& G_{n k}(r)=\frac{\Gamma^{2}(2 \gamma+1)}{\Gamma^{3 / 2}(n+2 \gamma+1)}(n !)^{3 / 2} \times \\
& \times \sqrt{2 \mathrm{n}+2 \gamma+1} r^{\gamma+1 / 2} e^{-\varepsilon r} L_{n}^{(2 \gamma)}(2 \varepsilon r)
\end{aligned}
$$

\section{NONCOMMUTATIVE RELATIVISTIC HAM- ILTONIAN FOR EXTENDED MODIFIED MIE-TYPE POTENTIAL}

\subsection{Formalism of Boopp's Shift Method}

Now, we shall review some fundamental principles of the quantum noncommutative Dirac equation which resumed in the following steps for modified Mie-type potential $V(\hat{r})[48,49,53,54,58,61]$ :

- Ordinary Hamiltonian $\hat{H}\left(p_{i}, x_{i}\right)$ replace by noncommutative Hamiltonian $\hat{H}\left(\hat{p}_{i}, \hat{x}_{i}\right)$,

- Ordinary spinor $\Psi(\vec{r})$ replace by new spinor $\hat{\Psi}(\overrightarrow{\hat{r}})$,

- Ordinary energy $\mathrm{E}_{\mathrm{nk}}$ replace by new energy $E_{n c-m t}$, and ordinary product replace by new star product $*$.

Which allow us to writing the noncommutative Dirac equation as follows:

$$
\hat{H}\left(\hat{p}_{i}, \hat{x}_{i}\right) * \widehat{\Psi}(\overrightarrow{\vec{r}})=E_{n c} \widehat{\Psi}(\overrightarrow{\vec{r}})
$$

The Boopp's shift method permutes to reduce the above equation to simplest the form:

$$
H_{n c-m t}\left(\hat{p}_{i}, \hat{x}_{i}\right) \psi(\vec{r})=E_{n c-m t} \psi(\vec{r})
$$

Where the new operator of Hamiltonian $H_{n c-m t}\left(\hat{p}_{i}, \hat{x}_{i}\right)$ can be expressed in three general varieties: both noncommutative space and noncommutative phase (NC-3D: RSP), only noncommutative space (NC3D: RS) and only noncommutative phase (NC: 3D-RP) as, respectively:

$$
\begin{aligned}
& H_{n c-m t}\left(\hat{p}_{i}, \hat{x}_{i}\right) \equiv H\left(\hat{p}_{i}=p_{i}-\frac{1}{2} \bar{\theta}_{i j} x_{j} ; \hat{x}_{i}=x_{i}-\frac{1}{2} \theta_{i j} p_{j}\right) \\
& \text { for NC-3D: RSP }
\end{aligned}
$$

$$
H_{n c-m t}\left(\hat{p}_{i}, \hat{x}_{i}\right) \equiv H\left(\hat{p}_{i}=p_{i} ; \hat{x}_{i}=x_{i}-\frac{1}{2} \theta_{i j} p_{j}\right)
$$

for NC-3D: RS

$$
\begin{aligned}
& H_{n c-m t}\left(\hat{p}_{i}, \hat{x}_{i}\right) \equiv H\left(\hat{p}_{i}=p_{i}-\frac{1}{2} \bar{\theta}_{i j} ; x_{j}, \hat{x}_{i}=x_{i}\right) \\
& \text { for NC-3D: RP }
\end{aligned}
$$

In recently work, we are interest with the first variety (19.2) and then the new modified Hamiltonian $H_{n c-m t}\left(\hat{p}_{i}, \hat{x}_{i}\right)$ defined as a function of $\hat{x}_{i}=x_{i}-\frac{1}{2} \theta_{i j} p_{j}$ and $\hat{p}_{i}=p_{i}$ :

$$
\begin{aligned}
& H_{n c-3 i s}\left(\hat{p}_{i}, \hat{x}_{i}\right)=\alpha \hat{\mathrm{P}}+\beta(M+S(\hat{r}))- \\
& -i \beta \hat{r} U(\hat{r}) \alpha+V(\hat{r})
\end{aligned}
$$

where the modified extended tensor interaction $U(\hat{r})$ and extended Mie-type potential $V(\hat{r})$ are given by, respectively:

$$
U(\hat{r})=-\frac{T}{\hat{r}}
$$

and

$$
V(\hat{r})=\frac{a}{\hat{r}^{2}}-\frac{b}{\hat{r}}+c
$$

The Dirac equation in the presence of above interactions $U(\hat{r})$ and $V(\hat{r})$ can be rewritten according Bop shift method as follows:

$$
\begin{aligned}
& (\alpha \mathrm{P}+\beta(M+S(\hat{r}))-i \beta \hat{r} U(\hat{r}) \alpha) \Psi(r, \theta, \phi)= \\
& =(E-V(\hat{r})) \Psi(r, \theta, \phi)
\end{aligned}
$$

The radial functions $\left(F_{n k}(r), G_{n k}(r)\right)$ are obtained, in the absence of tensor interaction, by solving two equations:

$$
\begin{aligned}
& {\left[\frac{d}{d r}+\frac{k}{r}\right] F_{n k}(r)=\left[M+E_{n c-m t}-\Delta(\hat{r})\right] G_{n k}(r)} \\
& {\left[\frac{d}{d r}+\frac{k}{r}\right] G_{n k}(r)=\left[M-E_{n c-m t}+\Sigma(\hat{r})\right] G_{n k}(r)}
\end{aligned}
$$

with $\Delta(\hat{r})=V(\hat{r})-S(\hat{r})$ and $\Sigma(\hat{r})=V(\hat{r})+S(\hat{r})$, eliminating $F_{n k}(r)$ and $G_{n k}(r)$ from Eqs. (24.1-2), we can obtain the following two Schrödinger-like differential equations as follows in NC-3D: RS:

$$
\begin{aligned}
& {\left[\frac{d^{2}}{d r^{2}}-\frac{k(k+1)}{r^{2}}-\left(M+E_{n c-m t}-\Delta(\hat{r})\right)\left(M-E_{n k}+\Sigma(\hat{r})\right)\right] \times{ }_{(2}} \\
& \times F_{n k}(r)=0
\end{aligned}
$$

$\left[\frac{d^{2}}{d r^{2}}-\frac{k(k-1)}{r^{2}}-\left(M+E_{n c-m t}-\Delta(\hat{r})\right)\left(M-E_{n k}+\Sigma(\hat{r})\right)\right] \times{ }_{2}$ $\times G_{n k}(r)=0$

After straightforward calculations one can obtains the different terms in (NC-3D: RS) spaces as follows:

$$
\begin{aligned}
& \frac{a}{\hat{r}^{2}}=\frac{a}{r^{2}}+\frac{a}{r^{4}} \overrightarrow{\mathbf{L}} \vec{\Theta}+O\left(\theta^{2}\right) \\
& -\frac{T}{\hat{r}}=\frac{T}{r}-\frac{T b}{2 r^{3}} \overrightarrow{\mathbf{L}} \vec{\Theta}+O\left(\theta^{2}\right) \\
& -\frac{b}{\hat{r}}=\frac{b}{r}-\frac{b}{2 r^{3}} \overrightarrow{\mathbf{L}} \vec{\Theta}+O\left(\theta^{2}\right)
\end{aligned}
$$


Which allow us to writing the modified extended tensor interaction $U(\hat{r})$ and extended Mie-type potential $V(\hat{r})$ as follows:

$$
\begin{aligned}
& U(\hat{r})=-\frac{T}{r}+U_{\text {pert }-m t}(r, \Theta) \\
& V(\hat{r})=\frac{a}{r^{2}}-\frac{b}{r}+c+\hat{V}_{\text {pert-mt }}(r, \Theta, a, b)
\end{aligned}
$$

with

$$
\hat{V}_{\text {pert-mt }}(r, \Theta, a, b)=\left(\frac{a}{r^{4}}-\frac{b}{2 r^{3}}\right) \overrightarrow{\mathbf{L}} \vec{\Theta}
$$

and

$$
U_{p e r t-m t}(r, \Theta, b)=-\frac{T b}{2 r^{3}} \overrightarrow{\mathbf{L}} \vec{\Theta}
$$

We generalized the constraint for the pseudospin (pspin) symmetry $(\Delta(r)=V(r) \quad$ and $\Sigma(r)=C_{p s}=$ constants which presented in ref. [36] into the new form $\Delta(\hat{r})=V(\hat{r})$ and $\Sigma(\hat{r})=\hat{C}_{p s}=$ constants in NC-3D: RS and inserting the potential $V(\hat{r})$ in eq. (27) into the two Schrödinger-like differential equations (25.1-2), one obtains:

$$
\begin{aligned}
& {\left[\begin{array}{l}
\frac{d^{2}}{d r^{2}}-\frac{k(k+1)}{r^{2}}-\left(M+E_{n c-m t}\right) \times \\
\times\left(M-E_{n c-m t}+C_{p s}\right)-\left(\frac{a}{r^{2}}-\frac{b}{r}+c\right) \times \\
\times\left(M-E_{n c-m t}+C_{p s}\right)- \\
-\left(\frac{a}{r^{4}}-\frac{b}{2 r^{3}}\right) \overrightarrow{\mathbf{L}} \vec{\Theta}\left(M-E_{n c-m t}+\hat{C}_{p s}\right)
\end{array}\right] F_{n k}(r)=0(29.1)} \\
& {\left[\begin{array}{l}
\frac{d^{2}}{d r^{2}}-\frac{k(k-1)}{r^{2}}-\left(M+E_{n c-m t}\right) \times \\
\times\left(M-E_{n k}+\hat{C}_{p s}\right)-\left(\frac{a}{r^{2}}-\frac{b}{r}+c\right) \times \\
\times\left(M-E_{n c-m t}+\hat{C}_{p s}\right)- \\
-\left(\frac{a}{r^{4}}-\frac{b}{2 r^{3}}\right) \overrightarrow{\mathbf{L}} \vec{\Theta}\left(M-E_{n c-m t}+\hat{C}_{p s}\right)
\end{array}\right] G_{n k}(r)=0(29.2)}
\end{aligned}
$$

It's clearly that, the additive new part $V_{\text {pert-mt }}(r, \Theta)$ is proportional with infinitesimal parameter $\Theta$, thus, we can considered as a perturbations terms.

4. THE EXACT RELATIVISTIC SPIN-ORBITAL HAMILTONIAN AND THE CORRESPONDING SPECTRUM FOR (M.M.T.) POTENTIAL IN (NC: 3D- RS) SYMMETRIES FOR EXCITED $n^{\text {th }}$ STATES FOR ONE-ELECTRON ATOMS

4.1 The Exact Relativistic Spin-orbital Hamiltonian for (m.m.t.) Potential in (NC: 3D- RS) Symmetries for One-electron Atoms

Again, the perturbative two terms $\hat{V}_{p e r t-m t}(r, \Theta, a, b)$ can be rewritten to the equivalent physical form for (m.m.t.) potential:

$$
\hat{V}_{\text {pert-mt }}(r, \Theta, a, b)=\left(\frac{a}{r^{4}}-\frac{b}{2 r^{3}}\right) \overrightarrow{\tilde{S}} \vec{L}
$$

Furthermore, the above perturbative terms $\hat{V}_{\text {pert-mt }}(r, \Theta, a, b)$ can be rewritten to the following new equivalent form for (m.m.t.) potential:

$$
\hat{V}_{p e r t-m t}(r, \Theta, a, b)=\frac{1}{2} \Theta\left(\frac{a}{r^{4}}-\frac{b}{2 r^{3}}\right)\left(\vec{J}^{2}-\vec{L}^{2}-\overrightarrow{\widetilde{S}}^{2}\right)
$$

To the best of our knowledge, we just replace the coupling spin-orbital $\vec{S} \vec{L}$ by the expres$\operatorname{sion} \frac{1}{2}\left(\vec{J}^{2}-\overleftrightarrow{L}^{2}-\overrightarrow{\widetilde{S}}^{2}\right)$, in relativistic quantum mechanics. The set $\left(H_{n c-m t}\left(\hat{p}_{i}, \hat{x}_{i}\right), \mathrm{J}^{2}, \mathrm{~L}^{2}, \tilde{\mathrm{S}}^{2}\right.$ and $\left.J_{z}\right)$ forms a complete of conserved physics quantities and the spinorbit quantum number $k(\tilde{k})$ is related to the quantum numbers for spin symmetry $l$ and p-spin symmetry $\tilde{l}$ as follows [36-37]:

$$
k=\left\{\begin{array}{l}
k_{1} \equiv-(l+1) \quad \text { if }-(\mathrm{j}+1 / 2),\left(\mathrm{s}_{1 / 2}, p_{3 / 2}, e t c\right), j=l+\frac{1}{2}, \\
\text { aligned spin }(\mathrm{k}\langle 0) \\
k_{2} \equiv+l \quad \text { if }\left(j=l+\frac{1}{2}\right),\left(\mathrm{p}_{1 / 2}, d_{3 / 2}, \text { etc }\right), j=l-\frac{1}{2}, \\
\text { unaligned spin }(\mathrm{k}>0)
\end{array}\right.
$$

and

$$
\tilde{k}=\left\{\begin{array}{l}
\tilde{k}_{1} \equiv-\tilde{l} \quad \text { if }-(\mathrm{j}+1 / 2),\left(\mathrm{s}_{1 / 2}, p_{3 / 2}, \text { etc }\right), j=\tilde{l}-\frac{1}{2}, \\
\text { aligned spin }(\mathrm{k}\langle 0) \\
\tilde{k}_{2} \equiv+(\tilde{l}+1) \quad \text { if }\left(j=\tilde{l}+\frac{1}{2}\right),\left(\mathrm{p}_{1 / 2}, d_{3 / 2}, \text { etc }\right), \\
\left.j=\tilde{l}+\frac{1}{2}, \text { unaligned spin }(\mathrm{k}\rangle 0\right)
\end{array}\right.
$$

With $\tilde{k}(\tilde{k}-1)=\tilde{l}(\tilde{l}+1)$ and $k(k-1)=l(l+1)$, which allows us to form two diagonal $(3 \times 3)$ matrixes $\hat{H}_{s o-m t}\left(k_{1}, k_{2}\right)$ and $\hat{\tilde{H}}_{s o-m t}\left(\tilde{k}_{1}, \tilde{k}_{2}\right)$, for (m.m.t.) potential, respectively, in (NC: 3D-RS) as:

$$
\begin{aligned}
& \left(\hat{H}_{s o-m t}\right)_{11}\left(k_{1}\right)=k_{1} \Theta\left(\frac{a}{r^{4}}-\frac{b}{2 r^{3}}\right) \text { if } \\
& -(\mathrm{j}+1 / 2),\left(\mathrm{s}_{1 / 2}, p_{3 / 2}, \text { etc }\right), j=l+\frac{1}{2},
\end{aligned}
$$

aligned spin $(\mathrm{k}<0)$ 


$$
\begin{gathered}
\left(\hat{H}_{s o-m t}\right)_{22}\left(k_{2}\right)=k_{2} \Theta\left(\frac{a}{r^{4}}-\frac{b}{2 r^{3}}\right) \text { if } \\
\left(j=l+\frac{1}{2}\right),\left(\mathrm{p}_{1 / 2}, d_{3 / 2}, \text { etc }\right), j=l-\frac{1}{2},
\end{gathered}
$$

unaligned spin $(\mathrm{k}>0)$

$\left(\hat{H}_{s o-m t}\right)_{33}=0$

and

$$
\begin{gathered}
\left(\hat{\tilde{H}}_{s o-m t}\right)_{11}\left(\tilde{k}_{1}\right)=\tilde{k}_{1} \Theta\left(\frac{a}{r^{4}}-\frac{b}{2 r^{3}}\right) \text { if } \\
-(\mathrm{j}+1 / 2),\left(\mathrm{s}_{1 / 2}, p_{3 / 2}, \text { etc }\right), j=\tilde{l}-\frac{1}{2},
\end{gathered}
$$

aligned $\operatorname{spin}(\mathrm{k}<0)$

$$
\begin{aligned}
& \left(\hat{\tilde{H}}_{s o-m t}\right)_{22}\left(\tilde{k}_{2}\right)=\tilde{k}_{2} \Theta\left(\frac{a}{r^{4}}-\frac{b}{2 r^{3}}\right) \quad \text { if } \\
& \left(j=\tilde{l}+\frac{1}{2}\right),\left(\mathrm{p}_{1 / 2}, d_{3 / 2}, \text { etc }\right), j=\tilde{l}+\frac{1}{2},
\end{aligned}
$$

unaligned spin $(\mathrm{k}>0)$

$$
\left(\hat{\tilde{H}}_{s o-m t}\right)_{33}=0
$$

4.2 The Exact Relativistic Spin-orbital Spectrum for (m.m.t.) Potential Symmetries for $n^{\text {th }}$ States for One-electron Atoms in (NC: 3DRSP)

In this sub section, we are going to study the modifications to the energy levels ( $\left.E_{n c-\text { per: }:}\left(\Theta, \tilde{k}_{1}\right), E_{n c-\text { per:u }}\left(\Theta, \tilde{k}_{2}\right)\right) \quad$ for $\quad(-(\mathrm{j}+1 / 2)$, $\left(\mathrm{s}_{1 / 2}, p_{3 / 2}, e t c\right), j=\tilde{l}+\frac{1}{2}$, aligned $\operatorname{spin} \mathrm{k}\langle 0$ and spindown $)$ and $\left(j=\tilde{l}+\frac{1}{2}, \quad\left(\mathrm{p}_{1 / 2}, d_{3 / 2}\right.\right.$, etc $), j=\tilde{l}-\frac{1}{2}, \quad$ un aligned spin $\mathrm{k}>0$ and spin up), respectively, at first order of infinitesimal parameter $\Theta$, for excited states $n^{\text {th }}$, obtained by applying the standard perturbation theory, using eqs. (12) and (31) as:

$$
\begin{aligned}
& E_{n c-p e r: d}\left(\Theta, \tilde{k}_{1}\right) \equiv 2 \Theta \tilde{k}_{1} \frac{\Gamma^{4}(2 k+2 T)}{\Gamma^{3}(n+2 k+2 T)}(n !)^{3}(\mathrm{n}+2 k+2 T) \\
& \int r^{2 k+2 T} e^{-2 \sqrt{M^{2}-E_{n k}^{2}} r}\left[L_{n}^{(2 k+2 T-1)}\left(2 \sqrt{M^{2}-E^{2}{ }_{n k}} r\right)\right]^{2}\left(\frac{a}{r^{4}}-\frac{b}{2 r^{3}}\right) r^{2} d r
\end{aligned}
$$

$$
\begin{aligned}
& E_{n c-p e r: u}\left(\Theta, \tilde{k}_{2}\right) \equiv 2 \Theta \tilde{k}_{2} \frac{\Gamma^{4}(2 k+2 T)}{\Gamma^{3}(n+2 k+2 T)}(n !)^{3}(\mathrm{n}+2 k+2 T) \\
& \int r^{2 k+2 T} e^{-2 \sqrt{M^{2}-E_{n k}^{2}} r}\left[L_{n}^{(2 k+2 T-1)}\left(2 \sqrt{M^{2}-E^{2}{ }_{n k}} r\right)\right]^{2}\left(\frac{a}{r^{4}}-\frac{b}{2 r^{3}}\right) r^{2} d r
\end{aligned}
$$

A direct simplification gives:

$$
\begin{aligned}
& E_{n c-p e r: d}\left(\Theta, \tilde{k}_{1}\right) \equiv 2 \Theta \tilde{k}_{1} \frac{\Gamma^{4}(2 k+2 T)}{\Gamma^{3}(n+2 k+2 T)}(n !)^{3} \times \\
& \times(\mathrm{n}+2 k+2 T)\left(T_{1-m t}+T_{2-m t}\right) \\
& E_{n c-p e r: u}\left(\Theta, \tilde{k}_{2}\right) \equiv 2 \Theta \tilde{k}_{2} \frac{\Gamma^{4}(2 k+2 T)}{\Gamma^{3}(n+2 k+2 T)}(n !)^{3} \times \\
& \times(\mathrm{n}+2 k+2 T)\left(T_{1-m t}+T_{2-m t}\right)
\end{aligned}
$$

Where, the two terms $T_{1-m t}$ and $T_{2-m t}$ are given by:

$$
\begin{aligned}
& T_{1-m t}=a \int_{0}^{+\infty} r^{2 k+2 T-2} e^{-2 \sqrt{M^{2}-E_{n k}^{2}} r}\left[L_{n}^{(2 k+2 T-1)}\left(2 \sqrt{M^{2}-E^{2}{ }_{n k}} r\right)\right]^{2} d r \\
& T_{2-m t}=-\frac{b}{2} \int_{0}^{+\infty} r^{2 k+2 T-1} e^{-2 \sqrt{M^{2}-E_{n k}^{2}}}\left[L_{n}^{(2 k+2 T-1)}\left(2 \sqrt{M^{2}-E_{n k}^{2}} r\right)\right]^{2} d r
\end{aligned}
$$

This can rewrite as $\left(2 \sqrt{M^{2}-E^{2}} r=X\right)$ :

$$
\begin{aligned}
& T_{1-m t}=\frac{a}{2}\left(M^{2}-E_{n k}^{2}\right)^{k+T-1 / 2} \int_{0}^{+\infty} X^{2 k+2 T-2} e^{-X}\left[L_{n}^{(2 k+2 T-1)}(X)\right]^{2} d X \\
& T_{2-m t}=-\frac{b}{4}\left(M^{2}-E_{n k}^{2}\right)^{k+T-1} \int_{0}^{+\infty} X^{2 k+2 T-1} e^{-X}\left[L_{n}^{(2 k+2 T-1)}(X)\right]^{2} d X
\end{aligned}
$$

Know we apply the special integral $[1,74]$ :

$$
\begin{aligned}
J_{n, \alpha}^{(\gamma)} & =\int_{0}^{\infty} e^{-x} x^{\alpha+\gamma}\left[L_{n}^{\alpha}(x)\right]^{2} d x \\
& =\frac{(\alpha+n) !}{n !} \sum_{i=0}^{n}(-1)^{i} \frac{\Gamma(n+i+\gamma)}{\Gamma(-i-\gamma)} \frac{(\alpha+i+\gamma) !}{(\alpha+i) !} \frac{1}{i !(n-i) !}
\end{aligned}
$$

$\operatorname{Re}(\alpha+\gamma+1)\rangle 0, \quad \gamma \quad$ can $\quad$ be takes: $(-1, \quad 0)$ and $\alpha \rightarrow 2 k+2 T-1$, which allow us to obtaining

$$
\begin{aligned}
& T_{1-m t}=\frac{a}{2}\left(M^{2}-E_{n k}^{2}\right)^{k+T-1 / 2} J_{n,(2 k+2 T-1)}^{(-1)} \\
& =\frac{a}{2}\left(M^{2}-E^{2}{ }_{n k}\right)^{k+T-1 / 2} \frac{(n+2 k+2 T-1) !}{n !} \times \\
& \times \sum_{i=0}^{n}(-1)^{i} \frac{\Gamma(n+i-1)}{\Gamma(1-i)} \frac{(2 k+2 T+i-2) !}{(2 k+2 T+i) !} \frac{1}{i !(n-i) !} \\
& T_{2-m t}=-\frac{b}{4}\left(M^{2}-E^{2}{ }_{n k}\right)^{k+T-1} J_{n,(2 k+2 T-1)}^{(0)} \\
& =-\frac{b}{4}\left(M^{2}-E^{2}{ }_{n k}\right)^{k+T-1} \frac{(n+2 k+T-1) !}{n !} \times \\
& \times \sum_{i=0}^{n}(-1)^{i} \frac{\Gamma(n+i+\gamma)}{\Gamma(-i)} \frac{(2 k+T-1+i) !}{(2 k+T+i-1) !} \frac{1}{i !(n-i) !}
\end{aligned}
$$

On the other hand, it is possible to obtain the term $T_{2-m t}$ by applying the following special integral [36, 75]:

$$
\int_{0}^{\infty} x^{q} e^{-x} L_{n}^{q}(x) L_{n^{\prime}}^{q}(x) d x=\frac{\Gamma(q+n+1)}{n !} \delta_{n n^{\prime}}
$$

Then, the term $T_{2-m t}$ is written as:

$$
T_{2-m t}=-\frac{b}{4}\left(M^{2}-E_{n k}^{2}\right)^{k+T-1} \frac{\Gamma(2 k+2 T+n)}{n !}
$$


Which allow us to obtaining the exact modifications of fundamental states $\left(E_{n c-p e r: d}\left(\Theta, \tilde{k}_{1}\right), E_{n c-p e r: u}\left(\Theta, \tilde{k}_{2}\right)\right)$ produced by relativistic spin-orbital effect:

$$
\begin{aligned}
& E_{n c-p e r: d}\left(\Theta, \tilde{k}_{1}\right) \equiv 2 \Theta \tilde{k}_{1} \frac{\Gamma^{4}\left(2 \tilde{k}_{1}+2 T\right)}{\Gamma^{3}\left(n+2 \tilde{k}_{1}+2 T\right)}(n !)^{3} \times \\
& \times\left(\mathrm{n}+2 \tilde{k}_{1}+2 T\right) \\
& \left(\begin{array}{ll}
\frac{a}{2}\left(M^{2}-E^{2}{ }_{n k}\right)^{\tilde{k}_{1}+T-1 / 2} & J_{n,\left(2 \tilde{k}_{1}+2 T-1\right)}^{(-1)} \\
-\frac{b}{4}\left(M^{2}-E_{n k}^{2}\right)^{k+T-1} & \frac{\Gamma\left(2 \tilde{k}_{1}+2 T+n\right)}{n !}
\end{array}\right) \\
& E_{n c-p e r: u}\left(\Theta, \tilde{k}_{2}\right) \equiv 2 \Theta \tilde{k}_{2} \frac{\Gamma^{4}\left(2 \tilde{k}_{2}+2 T\right)}{\Gamma^{3}\left(n+2 \tilde{k}_{2}+2 T\right)}(n !)^{3} \times \\
& \times\left(\mathrm{n}+2 \tilde{k}_{2}+2 T\right) \\
& \left(\begin{array}{l}
\frac{a}{2}\left(M^{2}-E_{n \tilde{k}_{2}}^{2}\right)^{\tilde{k}_{2}+T-1 / 2} J_{n,(2 k+2 T-1)}^{(-1)} \\
-\frac{b}{4}\left(M^{2}-E_{n k}^{2}\right)^{k+T-1} \frac{\Gamma\left(2 \tilde{k}_{1}+2 T+n\right)}{n !}
\end{array}\right)
\end{aligned}
$$

\subsection{The Exact Relativistic Magnetic Spectrum for (m.m.t.) Potential for Excited $n^{\text {th }}$ States for One-electron Atoms in (NC: 3D- RS) Symmetries}

Having obtained the exact modifications to the energy levels $\left(E_{n c-p e r: d}\left(\Theta, \tilde{k}_{1}\right), E_{n c-p e r: u}\left(\Theta, \tilde{k}_{2}\right)\right)$, for exit-

ed $n^{\text {th }}$ states, produced with relativistic spin-orbital induced by noncommutative spin-orbital Hamiltonian operator, we now consider another interested physically meaningful phenomena, which also produced from the perturbative terms of Mie-type potential related to the influence of an external uniform magnetic field, it's sufficient to apply the following two replacements to describing these phenomena:

$$
\left(\frac{a}{r^{4}}-\frac{b}{2 r^{3}}\right) \overrightarrow{\mathbf{L}} \vec{\Theta} \rightarrow \chi\left(\frac{a}{r^{4}}-\frac{b}{2 r^{3}}\right) \vec{B} \vec{L} \text { and } \Theta \rightarrow \chi B
$$

Here $\chi$ is infinitesimal real proportional's constants, and we choose the magnetic field $\vec{B}=B \vec{k}$, which allow us to introduce the modified new magnetic Hamiltonian $\hat{H}_{\text {mag-mt }}(r, a, b, \chi)$ in (NC: 3D-RS), as:

$$
\hat{H}_{\text {mag-mt }}(r, a, b, \chi)=\chi\left(\frac{a}{r^{4}}-\frac{b}{2 r^{3}}\right)(\vec{B} \vec{J}-\overrightarrow{\widetilde{S}} \vec{B})
$$

Here $(-\vec{S} \vec{B})$ denote to the ordinary Hamiltonian of Zeeman Effect. To obtain the exact noncommutative magnetic modifications of energy $E_{\text {mag-mt }}(\chi, n, \tilde{m}, a, b)$ for modified Mie-type potential, which produced automatically by the effect of $\hat{H}_{m-m t}(r, a, b, \chi)$, we make the following two simultaneously replacements:

$$
\tilde{k}_{1} \rightarrow \tilde{\mathrm{m}} \text { and } \quad \Theta \rightarrow \chi
$$

Then, the relativistic magnetic modification $E_{\text {mag-mt }}(\chi, n, \tilde{m}, a, b)$ corresponding $n^{\text {th }}$ excited states, in (NC-3D: RS) symmetries, can be determined from the following relation:

$$
\begin{aligned}
& E_{\text {mag-mt }}(\chi, n, \tilde{m}, a, b)=2 \chi \tilde{m} B \frac{\Gamma^{4}\left(2 \tilde{k}_{1}+2 T\right)}{\Gamma^{3}\left(n+2 \tilde{k}_{1}+2 T\right)} \times \\
& \times(n !)^{3}\left(\mathrm{n}+2 \tilde{k}_{1}+2 T\right) \\
& \left(\begin{array}{l}
\frac{a}{2}\left(M^{2}-E_{n k}^{2}\right)^{\tilde{k}_{1}+T-1 / 2} \\
J_{n,\left(2 \tilde{k}_{1}+2 T-1\right)}^{(-1)} \\
-\frac{b}{4}\left(M^{2}-E_{n k}^{2}\right)^{k+T-1} \frac{\Gamma\left(2 \tilde{k}_{1}+2 T+n\right)}{n !}
\end{array}\right)
\end{aligned}
$$

Where $\tilde{m}$ denote to the angular momentum quantum number, $-\tilde{l} \leq \tilde{m} \leq+\tilde{l}$, which allow us to fixing $(2 \tilde{l}+1)$ values for the orbital angular momentum quantum numbers.

\section{THE EXACT MODIFIED GLOBAL SPEC- TRUM FOR (M.M.T.) POTENTIAL IN (NC-3D: RS) SYMMETRIES FOR ONE-ELECTRON ATOMS}

Let us now resume the $n^{\text {th }}$ excited states eigenenergies

$$
\left(E_{n c-d}\left(\Theta, \tilde{k}_{1}, \chi, n, \tilde{m}, a, b\right)\right. \text {, }
$$
$\left.E_{n c r: u}\left(\Theta, \tilde{k}_{2}, \chi, n, \tilde{m}, a, b\right)\right)$ of modified Dirac equation corresponding for $\left(-(\mathrm{j}+1 / 2), \quad\left(\mathrm{s}_{1 / 2}, p_{3 / 2}\right.\right.$, etc $), j=\tilde{l}+\frac{1}{2}$, aligned spin $\mathrm{k}\langle 0$ and spin-down $)$ and $\left(j=\tilde{l}+\frac{1}{2}\right.$, $\left(\mathrm{p}_{1 / 2}, d_{3 / 2}, e t c\right), j=\tilde{l}-\frac{1}{2}$, un aligned spin $\mathrm{k}>0$ and spin up), respectively, at first order of parameter $\Theta$, for (m.m.t.) potential in (NC: 3D-RS), respectively, on based to the obtained new results (42.1), (42.2and (46), in addition to the original results (13) of energies in commutative space, we obtain the following original results:

$$
\begin{aligned}
& E_{n c-d}\left(\Theta, \tilde{k}_{1}, \chi, n, \tilde{m}, a, b\right)=E_{n \tilde{k}_{1}}+\frac{2 \Gamma^{4}\left(2 \tilde{k}_{1}+2 T\right)}{\Gamma^{3}\left(n+2 \tilde{k}_{1}+2 T\right)} \times \\
& \times(n !)^{3}\left(\mathrm{n}+2 \tilde{k}_{1}+2 T\right) \\
& \left(\begin{array}{l}
\frac{a}{2}\left(M^{2}-E_{n \tilde{k}_{1}}^{2}\right)^{\tilde{k}_{1}+T-1 / 2} J_{n,\left(2 \tilde{k}_{1}+2 T-1\right)}^{(-1)} \\
-\frac{b}{4}\left(M^{2}-E_{n k}^{2}\right)^{k+T-1} \frac{\Gamma\left(2 \tilde{k}_{1}+2 T+n\right)}{n !}
\end{array}\right)\left\{\Theta \tilde{k}_{1}+\chi \tilde{m} B\right\}
\end{aligned}
$$


$E_{n c-u}\left(\Theta, \tilde{k}_{2}, \chi, n, \tilde{m}, a, b\right)=E_{n \tilde{k}_{2}}+\frac{2 \Gamma^{4}\left(2 \tilde{k}_{2}+2 T\right)}{\Gamma^{3}\left(n+2 \tilde{k}_{2}+2 T\right)} \times$

$\times(n !)^{3}\left(\mathrm{n}+2 \tilde{k}_{2}+2 T\right)$

$\left(\begin{array}{ll}\frac{a}{2}\left(M^{2}-E_{n \tilde{k}_{2}}^{2}\right)^{\tilde{k}_{2}+T-1 / 2} & J_{n,\left(2 \tilde{k}_{2}+2 T-1\right)}^{(-1)} \\ -\frac{b}{4}\left(M^{2}-E_{n \tilde{k}_{2}}^{2}\right)^{k+T-1} \frac{\Gamma\left(2 \tilde{k}_{2}+2 T+n\right)}{n !}\end{array}\right)\left\{\Theta \tilde{k}_{2}+\chi \tilde{m} B\right\}$

As it is montionated in ref. [36], in view of exact spin symmetry in commutative space $\left(E_{n k} \rightarrow-E_{n k}\right.$, $V(r) \rightarrow-V(r), \quad k \rightarrow k+1$ and $\left.G_{n \tilde{k}}(r) \rightarrow G_{n k}(r)\right)$, they take the following forms:

$$
b\left(E_{n k}-M\right)=2(k+T+n) \sqrt{M^{2}-E^{2}{ }_{n k}}
$$

We need to generalize the above translations to the case of noncommutative three dimensional spaces as:

$$
\begin{aligned}
& E_{n c-d}\left(\Theta, \tilde{k}_{1}, \chi, n, \tilde{m}, a, b\right) \rightarrow E_{n c-d}\left(\Theta, k_{1}, \chi, n, m, a, b\right) \\
& \equiv-E_{n c-d}\left(\Theta, \tilde{k}_{1}+1, \chi, n, m, a, b\right) \\
& E_{n c-u}\left(\Theta, \tilde{k}_{1}, \chi, n, \tilde{m}, a, b\right) \rightarrow E_{n c-u}\left(\Theta, k_{1}, \chi, n, m, a, b\right) \\
& \equiv-E_{n c-u}\left(\Theta, \tilde{k}_{2}+1, \chi, n, m, a, b\right) \\
& V(\hat{r}) \rightarrow-V(\hat{r}) \\
& \tilde{k}_{1} \rightarrow k_{1}+1 \quad \text { and } \quad \tilde{k}_{2} \rightarrow k_{2}+1
\end{aligned}
$$

It's clearly, that the obtained eigenvalues of energies are real and then the noncommutative Hamiltonian operator $\hat{H}_{n c-m t}$ is Hermitian,

$$
\begin{aligned}
& \hat{H}_{n c-m t}=\alpha \mathrm{P}+\beta(M+S(\hat{r}))-i \beta \hat{r} U(\hat{r}) \alpha+V(\hat{r})+ \\
& \Theta\left(\frac{a}{r^{4}}-\frac{b}{2 r^{3}}\right) \vec{S} \vec{L}+\chi\left(\frac{a}{r^{4}}-\frac{b}{2 r^{3}}\right)(\vec{B} \vec{J}-\overrightarrow{\widetilde{S}} \vec{B})
\end{aligned}
$$

In this way, one can obtain the complete energy spectra for (m.m.t.) potential in (NC: 3D-RS) symmetries. Know the following accompanying constraint relations:

1. The original spectrum contain two possible values of energies in ordinary three dimensional space which presented by equations (13) and (47),

2. The quantum number $\tilde{m}$ satisfied the interval: $-\tilde{l} \leq \tilde{m} \leq+\tilde{l}$, thus we have $(2 \tilde{l}+1)$ values for this quantum number,

3. We have also two values for $j=\tilde{l}+\frac{1}{2}$ and $j=\tilde{l}-\frac{1}{2}$.

4. Allow us to deduce the important original results: every state in usually three dimensional space will be replace by $2(2 \tilde{l}+1)$ sub-states and then the degenerated state can be take $2 \sum_{i=0}^{n-1}(2 l+1) \equiv 2 n^{2}$ values in (NC: 3D-RS) symmetries.

Know, we can apply the obtained results $(45,1-2)$ to the case of the modified Krazer-Futes potential $V_{K F}(\hat{r})$ as a simple example from the (m.m.t.) potential by setting $\left(a=D_{e} r_{e}^{2}, b=2 D_{e} r_{e}, c=0\right)$ :

$$
V_{K F}(\hat{r})=V_{K F}(r)+\hat{V}_{p e r t-K F}(r, \Theta, a, b)
$$

with

$$
\begin{gathered}
V_{K F}(r)=-D_{e}\left(\frac{2 r_{e}}{r}-\frac{r_{e}^{2}}{r^{2}}\right) \\
\text { and } \hat{V}_{p e r t-K F}(r, \Theta, a, b)=\left(\frac{D_{e} r_{e}^{2}}{r^{4}}-\frac{2 D_{e} r_{e}}{2 r^{3}}\right) \overrightarrow{\mathbf{L}} \vec{\Theta}
\end{gathered}
$$

$V_{K F}(r), D_{e}$ and $r_{e}$ are Krazer-Futes potential in commutative spaces, the dissociation energy and the equilibrium inter-nuclear length [36], thus the energy equations $(47,1-2)$ becomes:

$$
\begin{aligned}
& E_{n c-d}\left(\Theta, \tilde{k}_{1}, \chi, n, \tilde{m}\right)=E_{n \tilde{k}_{1}}+\frac{2 \Gamma^{4}\left(2 \tilde{k}_{1}+2 T\right)}{\Gamma^{3}\left(n+2 \tilde{k}_{1}+2 T\right)} \times \\
& \times(n !)^{3}\left(\mathrm{n}+2 \tilde{k}_{1}+2 T\right) \\
& \left(\begin{array}{l}
\frac{D_{e} r_{e}^{2}}{2}\left(M^{2}-E^{2}{ }_{n \tilde{k}_{1}}\right)^{\tilde{k}_{1}+T-1 / 2} J_{n,\left(2 \tilde{k}_{1}+2 T-1\right)}^{(-1)} \\
-\frac{D_{e} r_{e}}{2}\left(M^{2}-E_{n k}^{2}\right)^{k+T-1} \frac{\Gamma\left(2 \tilde{k}_{1}+2 T+n\right)}{n !}
\end{array}\right)\left\{\Theta \tilde{k}_{1}+\chi \tilde{m} B\right\}
\end{aligned}
$$

$$
\begin{aligned}
& E_{n c-u}\left(\Theta, \tilde{k}_{2}, \chi, n, \tilde{m}\right)=E_{n \tilde{k}_{2}}+\frac{2 \Gamma^{4}\left(2 \tilde{k}_{2}+2 T\right)}{\Gamma^{3}\left(n+2 \tilde{k}_{2}+2 T\right)} \times \\
& \times(n !)^{3}\left(\mathrm{n}+2 \tilde{k}_{2}+2 T\right) \\
& \left(\begin{array}{c}
\frac{D_{e} r_{e}^{2}}{2}\left(M^{2}-E^{2}{ }_{n \tilde{k}_{2}}\right)^{\tilde{k}_{2}+T-1 / 2} \\
J_{n,\left(2 \tilde{k}_{2}+2 T-1\right)}^{(-1)} \\
-\frac{D_{e} r_{e}}{2}\left(M^{2}-E_{n k}^{2}\right)^{k+T-1} \frac{\Gamma\left(2 \tilde{k}_{1}+2 T+n\right)}{n !}
\end{array}\right)\left\{\Theta \tilde{k}_{2}+\chi \tilde{m} B\right\}
\end{aligned}
$$

And the energy equation $E_{K F}\left(E_{n \tilde{k}_{1}}\right.$ and $\left.E_{n \tilde{k}_{2}}\right)$ for Krazer-Futes potential in commutative spaces by is given [36]:

$$
\begin{aligned}
& \frac{2 D_{e} r_{e}\left(E_{K F}-M\right)}{\sqrt{M^{2}-E_{K F}^{2}}}= \\
& =2 \sqrt{(k-1 / 2)^{2}+D_{e} r_{e}^{2}\left(E_{K F_{1}}-M\right)}+n+1 / 2
\end{aligned}
$$

\section{CONCLUSION}

In this study we have performed the exact analytical bound state solutions: the energy spectra and the corresponding noncommutative Hermitian Hamiltonian operator for three dimensional Dirac equations in spherical coordinates for (m.m.t.) potential by using generalization Boopp's Shift method and standard per- 
turbation theory. It is found that the energy eigenvalues depend on the dimensionality of the problem and new atomic quantum numbers $(j=\tilde{l} \pm 1 / 2, j=l \pm 1 / 2, \tilde{s}= \pm 1 / 2, l, \tilde{l})$ and the angular momentum quantum number in addition to the infinitesimal parameter $\Theta$ in the symmetries of (NC: 3DRSP). And we also showed that the obtained energy spectra degenerate and every old state will be replaced

\section{REFERENCES}

1. S.H. Dong, G.H. Sun, Phys. Scripta 70 (2-3), 94 (2004).

2. S.K. Bose, Il Nuovo Cimento B, 109 (11), 1217 (1994).

3. L. Buragohain, S. A. S. Ahmed, Lat. Am. J. Phys. Educ. 4 (1), 79 (2010).

4. A.N. Ikot, Afr. Rev Phys. 6, 221 (2011).

5. A. Arda, J. Math. Chem. 50, 971 (2012).

6. S.M. Ikhdair, B.J. Falaye, M. Hamzavi, Ann. Phys.-New York. 353, 282 (2015)

7. S.M. Ikhdair, R. Sever, J. Mol. Struc.-Theochem. 806, 155 (2007).

8. A.S. Ahmed, L. Buragohain, Phys.Scr. 80, 015701 (2009).

9. S.K. Bose, Nouvo Cimento B. 113, 299 (1998).

10. G.P. Flesses, A. Watt, J. Phys. A: Math. Gen. 14, 315 (1981).

11. M. Ikhdair, R. Sever, Ann. Phys. 16, 218 (2007).

12. S.H. Dong, Phys. Scripta. 64, 273 (2001).

13. S.H. Dong, Z.Q. Ma, J. Phys. A. Math. Gen. 31, 9855 (1998).

14. S.H. Dong, Int. J. Theor. Phys. 40, 559 (2001).

15. A. Akder, J. Theor. Appl. Physics. 7, 17 (2013).

16. B.I. Ita, A.I. Ikeuda, Journal of Atomic and Molecular Physics, 201, 4 (2013).

17. S.H. Dong, G.H. San, Found. Phys. Lett. 16, 357 (2003).

18. B.I. Ita, A.I. Ikeuba, A.N. Ikot, Commun. Theor. Phys. 61, 149 (2014).

19. H. Hassanabadi, M. Hamzavi, S. Zarrinkamar, A.A. Rajabi, Int. J. Phys. Sci. 6, 583 (2011).

20. S.H. Dong, Z.Q. Ma, G. Esposito, Found. Phys. Lett. 12, 465 (1999).

21. D. Agboola, Acta Phys. Pol. A. 120, 371 (2011).

22. D. Shi-Hai, Int. J. Theor. Phys. 39, 1119 (2000).

23. B.I. Ita, Int. J. Rec. Adv. Phys. 2 (4), (2013).

24. T. Das, A. Arda, Advances in High Energy PhysicsHindawi Publishing Corporation 2015, 8 (2015).

25. S.M. Ikhdair, R. Sever, Cent. Eur. J. Phys. 5 (4), 516 (2007).

26. S.M. Ikhdair, J. Mod. Phys. 3 (2), 170 (2012).

27. S.M. Ikhdair, R. Sever, Appl. Math. Comput. 216, 911 (2010).

28. S.M. Ikhdair, J. Math. Phys. 51 (2), 023525 (2010).

29. J.Y. Gou, Z.Q. Sheng, Phys. Lett. A 338, 90 (2005).

30. A. Arda, R. Sever, Int. J. Theor. Phys. 48, 945 (2009).

31. Y. Xu, S. He, C.S. Jia, J. Phys. A: Math. Theor. 41, 255302 (2008).

32. V.I. Kukulin, G. Loyola, M. Moshinsky, Phys. Lett. A 158, 19 (1991).

33. G. Mao, Phys. Rev. C 67, 044312 (2003).

34. A.D. Alhaidari, H. Bahlouli, A. Al-Hasan, Phys. Lett. A 349, 87 (2006).

35. R. Lisboa, M. Malheiro, A.S. De Castro, P. Alberto, M. by $2(2 \tilde{l}+1)$ sub-states. And we have also applied our obtained results to the case of modified Krazer-Futes potential.

\section{AKNOWLEDGEMENTS}

This work was supported with search laboratory of: Physics and Material Chemistry, in Physics department, Sciences faculty-University of M'sila, Algeria.

Fiolhais, Phys. Rev. C 69, 024315 (2004)

36. O. Aydogdu, R. Server, Ann. Phys. New. York 325, 373 (2010).

37. M. Eshghi, S.M. Ikhdair, Chin. Phys. B. 23 (12), 120304 (2014). 38. H. Snyder, Phys. Rev. 71, 38 (1946).

39. A. Maireche, Life. Sci. J. 11, 353 (2014).

40. A. Maireche, Afr. Rev. Phys. 9, 479 (2014).

41. A. Maireche, J. Nano- Electron. Phys. 7, 02003 (2015).

42. A. Maireche, Afr. Rev Phys. 9, 185 (2014).

43. A. Maireche, Afr. Rev Phys. 10, 97 (2015).

44. A. Maireche, Int. Lett. Chem. Phys. Astr. 56, 1 (2015).

45. A. Maireche, Int. Lett. Chem. Phys. Astr. 60, 11 (2015).

46. A. Maireche, Afr. Rev. Phys. 10, 177 (2015).

47. A. Maireche, Afr. Rev. Phys., 10, 373 (2015).

48. A. Maireche, Int. Lett. Chem. Phys. Astr. 58, 164 (2015).

49. A. Maireche, Med. J. Model. Simul. 4, 60 (2015).

50. A. Maireche, Int. Lett. Chem. Phys. Astr. 61, 38 (2015).

51. A. Maireche, J. Nano- Electron. Phys. 7, 04021 (2015).

52. A. Maireche, J. Nano- Electron. Phys. 7, 03047 (2015).

53. A. Maireche, Lat. Am. J. Phys. Educ. 9, 1301 (2015).

54. A. Maireche, Nano World J. 1, 120 (2016).

55. A. Maireche, J. Nano- Electron. Phys. 8, 01020 (2016).

56. A. Maireche, J. Nano- Electron. Phys. 8, 01021 (2016).

57. A. Maireche, J. Nano- Electron. Phys. 8, 02027 (2016).

58. A. Maireche, J. Nano-Electron. Phys. 8, 02046 (2016).

59. A.E.F. Djemei, H. Smail, Commun. Theor. Phys. 41, 837 (2004).

60. S. Cai, T. Jing, Int. J. Theor. Phys. 49, 1699 (2010).

61. Joohan Lee, J. Korean. Phys. Soc. 47, 571 (2005).

62. A. Jahan, Braz. J. Phys. 37, 144 (2007).

63. A.F. Dossa, G.Y.H. Avossevou, J. Mod. Phys. 41400 (2013).

64. Yang, Zu-Hua, Int. J. Theor. Phys. 49, 644 (2010).

65. Y. Yuan, Chinese Phys. C, 34, 543 (2010).

66. J. Mamat, S. Dulat, H. Mamatabdulla, Int J Theor Phys. 55, 2913 (2016).

67. B. Mirza, Commun. Theor. Phys. 55, 405 (2011).

68. Y. Xiao, Z. Long, S. Cai, Int. J. Theor. Phys. 50, 3105 (2011).

69. A.E.F. Djemaï, H. Smail, Commun. Theor. Phys. 41, 837 (2004).

70. A. Al-Jamel, J. Theor. Appl. Phys. 5, 21 (2011).

71. H. Hassanabadi, Eur. Phys. J. Plus. 130, 200 (2015).

72. Won sang Chung. Int. J. Theor. Phys. 54, 1840 (2015).

73. T.E. Perez, M.A. Pinnar, J. Approx. Theory 86, 278 (1996).

74. M.M. Nieto, L.M. Simmons, Phys. Rev. A 19, 438 (1979).

75. M. Abramowitz, I.A. Stegun, Handbook of Mathematical Functions with Formulas, Graphs, and Mathematical Tables

(Dover Publications, New York, 1965). 